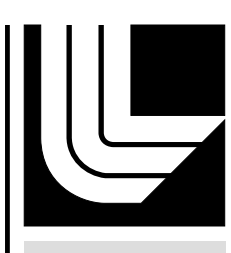

LAWRENCE LIVERMORE NATIONAL LABORATORY

Adaptive Particle Filtering for Mode Tracking: A Shallow Ocean Application

J. V. Candy

July 20, 2011

OCEANS'11

Kona, HI, United States

September 19, 2011 through September 22, 2011 
This document was prepared as an account of work sponsored by an agency of the United States government. Neither the United States government nor Lawrence Livermore National Security, LLC, nor any of their employees makes any warranty, expressed or implied, or assumes any legal liability or responsibility for the accuracy, completeness, or usefulness of any information, apparatus, product, or process disclosed, or represents that its use would not infringe privately owned rights. Reference herein to any specific commercial product, process, or service by trade name, trademark, manufacturer, or otherwise does not necessarily constitute or imply its endorsement, recommendation, or favoring by the United States government or Lawrence Livermore National Security, LLC. The views and opinions of authors expressed herein do not necessarily state or reflect those of the United States government or Lawrence Livermore National Security, LLC, and shall not be used for advertising or product endorsement purposes. 


\title{
Adaptive Particle Filtering for Mode Tracking: A Shallow Ocean Application
}

\author{
J. V. Candy, Fellow, IEEE
}

\begin{abstract}
The shallow ocean is an ever changing environment primarily due to temperature variations in its upper layers $(<100$ m) directly affecting sound propagation throughout. The need to develop processors capable of tracking these changes implies a stochastic as well as an environmentally adaptive design. The stochastic requirement follows directly from the multitude of variations created by uncertain parameters and noise. Some work has been accomplished in this area, but the stochastic nature was constrained to Gaussian uncertainties. It has been clear for a long time that this constraint was not particularly realistic leading to a Bayesian approach that enables the representation of any uncertainty distribution. Sequential Bayesian techniques enable a class of processors capable of performing in an uncertain, nonstationary (varying statistics), non-Gaussian, variable shallow ocean environment. A solution to this problem is addressed by developing a sequential Bayesian processor capable of providing a joint solution to the modal function tracking (estimation) and environmental adaptivity problem. The posterior distribution required is multi-modal (multiple peaks) requiring a sequential (nonstationary) Bayesian approach. Here the focus is on the development of a particle filter (PF) capable of providing reasonable performance for this problem. In our previous effort on this problem nonlinear/non-Gaussian processors were developed to operate on synthesized data based on the Hudson Canyon experiment using normal-mode representations. Here we extend the processors by applying them to the actual hydrophone measurements obtained from the 23 -element vertical array. The adaptivity problem is attacked by allowing the modal coefficients to be estimated from the measurement data jointly along with tracking of the modal functions-the main objective.

Index Terms - model-based processor, sequential Bayesian processor, sequential Monte Carlo, particle filter, Hudson Canyon experiment.
\end{abstract}

\section{INTRODUCTION}

The shallow ocean is an uncertain, ever changing, dispersive environment dominated by ambient and shipping noise as well as temperature fluctuations that alter sound propagation throughout. Environmental variations are created by these fluctuations (sound speed variations) as well as other disturbances caused by ambient, shipping and surface noise and sensor noise like flow and instrumentation noise. Modeling parametric uncertainties can also affect the processing problem. Thus, a processor is required to "adapt to these environmental variations while simultaneously tracking modal functions that are necessary for such applications as localization, inversion and signal enhancement. A possible solution to this problem is accomplished by developing a sequential Bayesian processor capable of providing a joint solution to the modal function tracking (estimation) and environmental adaptivity problem. The posterior distribution required is multi-modal (multiple peaks) requiring a sequential Bayesian approach.
One possible approach to this problem is called modelbased, that is, incorporating a propagation model into a signal processing scheme has evolved over a long period of time where it was recognized that by embedding a physics-based representation can significantly improve the processing [1][5]. In ocean acoustics there are many problems of interest [6]-[15] governed by propagation models of varying degrees of sophistication. Thus, we are interested in a shallow water environment characterized by a normal-mode model.

Model-based signal processing is concerned with the incorporation of environmental (propagation, seabed, sound speed, etc.), measurement (sensor arrays) and noise (ambient, shipping, surface, etc.) models along with measured data into a sophisticated processing algorithm capable of detecting, filtering (estimating) and localizing an acoustic source (target) in the complex ocean environment as well as adaptively estimating the model parameters themselves. These techniques are not constrained to a stationary environment which is essential in the ever changing ocean. Not only does the model-based approach offer a means of estimating various quantities of high interest, but it also provides a methodology to statistically evaluate its performance on-line [16]. Model-based techniques have been around for quite a while and have found their way into ocean acoustics [15].

In this paper, we are primarily interested in investigating the application of the so-called "next generation" of modelbased signal processing algorithms, primarily the unscented Kalman filter $(U K F)$ and the particle filter $(P F)$ with the goal of analyzing their performance on pressure-field data obtained from the well-known Hudson Canyon experiments performed on the New Jersey shelf [11], [12]. A $P F$ is a sequential Markov chain Monte Carlo (MCMC) Bayesian processor capable of providing reasonable performance for a multimodal problem estimating a non-parametric representation of the posterior distribution [23]. On the other hand, the UKF is a unimodal processor capable of representing any single peaked distribution using a statistical linearization technique based on sigma points that deterministically characterize the posterior [23].

Model-based processing is a direct approach that uses in-situ measurements. More specifically, the acoustic measurements are combined with a set of model parameters usually obtained from a priori information or a sophisticated normal-mode simulator [13] that solves the underlying boundary value problem to extract the initial parameters/states in order to construct the forward propagator and initialize the algorithm. The algorithm then uses the incoming data to update the parameter set jointly 
with the acoustic signal processing task (enhancement). In the following, we define a processor whose enhanced states are the estimated modal functions. Here we investigate the development of a "model-based signal enhancer" that embeds a forward propagator into the processing scheme essentially mimicking previous model-based efforts that used a class of linearized processors (linearized and extended Kalman filters (LZKF, EKF) [16], [17], [23]. In order to construct the modelbased processor $(B P)$, we first characterize the normal-mode model in terms of a state-space representation enabling a general framework for signal processing.

Background for the state-space representation of our problem is given in Section II leading to the formulation of the forward propagators. The design of the $B P$ for a shallow ocean acoustic problem is discussed in Section III and the results are given where we compare the performance of the $P F$ and $U K F$. We discuss our results in the final section.

\section{STATE-SPACE PROPAGATOR}

For our ocean acoustic modal function enhancement problem, we assume a horizontally-stratified ocean of depth $h$ with a known horizontal source range $r_{s}$ and depth $z_{s}$ and that the acoustic energy from a point source can be modeled as a trapped wave governed by the Helmholtz equation [9], [14]. The standard separation of variables technique and removing the time dependence leads to a set of ordinary differential equations, that is, we obtain a "depth only" representation of the wave equation which is an eigenvalue equation in $z$ with

$$
\frac{d^{2}}{d z^{2}} \phi_{m}(z)+\kappa_{z}^{2}(m) \phi_{m}(z)=0, m=1, \cdots, M
$$

whose eigensolutions $\left\{\phi_{m}(z)\right\}$ are the so called modal functions and $\kappa_{z}$ is the wave number in the z-direction. These solutions depend on the sound speed profile, $c(z)$, and the boundary conditions at the surface and bottom as well as the corresponding dispersion relation given by

$$
\kappa^{2}=\frac{\omega^{2}}{c^{2}(z)}=\kappa_{r}^{2}(m)+\kappa_{z}^{2}(m), \quad m=1, \ldots, M
$$

where $\kappa_{r}(m)$ is the horizontal wave number associated with the $m$-th mode in the $r$ direction and $\omega$ is the harmonic source frequency.

By assuming a known horizontal source range a priori, we obtain a range solution given by the Hankel function, $H_{0}\left(\kappa_{r} r_{s}\right)$ enabling the pressure-field to be represented by

$$
p\left(r_{s}, z\right)=\sum_{m=1}^{M} \beta_{m}\left(r_{s}, z_{s}\right) \phi_{m}(z)
$$

where $p$ is the acoustic pressure; $\phi_{m}$ is the $m^{t h}$ modal function with the modal coefficient defined by

$$
\beta_{m}\left(r_{s}, z_{s}\right):=q H_{0}\left(\kappa_{r} r_{s}\right) \phi_{m}\left(z_{s}\right)
$$

for $q$ is the source amplitude.

\section{A. State-Space Model}

The depth-only eigen-equation can easily be transformed to state-space form by defining the state vector of the $m$-th mode as

$$
\phi_{m}(z):=\left[\begin{array}{c}
\phi_{m}(z) \\
\frac{d}{d z} \phi_{m}(z)
\end{array}\right]=\left[\begin{array}{c}
\phi_{m 1}(z) \\
\phi_{m 2}(z)
\end{array}\right]
$$

Thus, we have for the $m$-th mode the following state (vector) equation as:

$$
\frac{d}{d z} \phi_{m}(z)=\mathbf{A}_{m}(z) \phi_{m}(z)
$$

for

$$
\mathbf{A}_{m}(z)=\left[\begin{array}{cc}
0 & 1 \\
-\kappa_{z}^{2}(m) & 0
\end{array}\right]
$$

Assuming that the ocean acoustic noise can be characterized by additive uncertainties, we can extend the deterministic state equation for the $M$-modes, that is, $\Phi(z):=$ $\left[\phi_{1}(z)|\cdots| \phi_{M}(z)\right]^{T}$ leading to the following $2 M$-dimensional Gauss-Markov representation of the model:

$$
\frac{d}{d z} \phi(z)=\mathbf{A}(z) \phi(z)+\mathbf{w}(z)
$$

where $\mathbf{w}(z)=\left[\begin{array}{llll}w_{1} & w_{2} & \ldots & w_{2 M}\end{array}\right]^{T}$ is additive, zero-mean random noise. The system matrix $\mathbf{A}(z)$ is defined as

$$
\mathbf{A}(z)=\left[\begin{array}{ccc}
\mathbf{A}_{1}(z) & \cdots & 0 \\
\vdots & \ddots & \vdots \\
0 & \cdots & \mathbf{A}_{M}(z)
\end{array}\right]
$$

and the overall state vector is

$$
\phi(z)=\left[\begin{array}{llllllll}
\phi_{11} & \phi_{12} \mid \phi_{21} & \phi_{22} & \ldots & \phi_{M 1} & \phi_{M 2}
\end{array}\right]^{T}
$$

This leads to the measurement equations, which we can write as

$$
p\left(r_{s}, z\right)=\mathbf{C}^{T}\left(r_{s}, z_{s}\right) \phi(z)+v(z)
$$

where

$$
\mathbf{C}^{T}\left(r_{s}, z_{s}\right)=\left[\begin{array}{llllll}
\beta_{1}\left(r_{s}, z_{s}\right) & 0 & \cdots & \mid \beta_{M}\left(r_{s}, z_{s}\right) & 0
\end{array}\right]
$$

The random noise terms $\mathbf{w}(z)$ and $v(z)$ can be assumed Gaussian and zero-mean with respective covariance matrices, $\mathbf{R}_{w w}$ and $\mathbf{R}_{v v}$. The measurement noise $(v(z))$ can be used to represent the "lumped" effects of near-field acoustic noise field, flow noise on the hydrophone and electronic noise. The modal noise $(\mathbf{w}(z))$ can be used to represent the "lumped" uncertainty of sound speed errors, distant shipping noise, errors in the boundary conditions, sea state effects and ocean inhomogeneities that propagate through the ocean acoustic system dynamics (normal-mode model). These assumptions, with known model parameters lead to the optimal solution of the state estimation problem (Kalman filter) [17]. 
Since the array spatially samples the pressure-field discretizing depth, we choose to analogously discretize the differential state equations using a central difference approach for improved numerical stability, that is, from Eq. 1 we have

$$
\frac{d^{2}}{d z^{2}} \phi_{m} \approx \frac{\phi_{m}\left(z_{\ell}\right)-2 \phi_{m}\left(z_{\ell-1}\right)+\phi_{m}\left(z_{\ell-2}\right)}{\triangle z_{\ell}^{2}}
$$

for $\triangle z_{\ell}:=z_{\ell}-z_{\ell-1}$. Applying this approximation to Eq. 1 gives

$\phi_{m}\left(z_{\ell}\right)-2 \phi_{m}\left(z_{\ell-1}\right)+\phi_{m}\left(z_{\ell-2}\right)+\triangle z_{\ell}^{2} \kappa_{z}^{2}(m) \phi_{m}\left(z_{\ell-1}\right)=0$

where $z_{\ell}$ is the location of the $\ell$-th sensor. Defining the discrete modal state vector as $\phi_{m}\left(z_{\ell}\right):=\left[\phi_{m}\left(z_{\ell-2}\right) \mid \phi_{m}\left(z_{\ell-1}\right)\right]^{T}$, we obtain the following set of difference equations for the $m$-th mode

$$
\begin{aligned}
& \phi_{m 1}\left(z_{\ell}\right)=\phi_{m 2}\left(z_{\ell-1}\right) \\
& \phi_{m 2}\left(z_{\ell}\right)=-\phi_{m 1}\left(z_{\ell-1}\right)+\left(2-\triangle z_{\ell}^{2} \kappa_{z}^{2}(m)\right) \phi_{m 2}\left(z_{\ell-1}\right)
\end{aligned}
$$

with each of the corresponding $A$-submatrices given by

$$
\mathbf{A}_{m}(z)=\left[\begin{array}{cc}
0 & 1 \\
-1 & 2-\triangle z_{\ell}^{2} \kappa_{z}^{2}(m)
\end{array}\right] ; \quad m=1, \cdots, M
$$

\section{B. Augmented State-Space Model}

The "parametrically adaptive" processor evolves from this representation by defining a parameter set of interest. Since we are primarily interested in an environmentally adaptive processor, that is, a processor capable of adjusting its parameters to variations in the environment such as temperature, noise, etc. We choose to capture these changes by allowing the modal coefficients to vary. Therefore, we define the parameter vector as

$$
\theta_{m}\left(r_{s}, z_{s}\right):=\beta_{m}\left(r_{s}, z_{s}\right) ; m=1, \cdots, M
$$

and a new "augmented" state vector as

$$
\Phi_{m}\left(z_{\ell} ; \theta_{m}\right):=\Phi_{m}\left(z_{\ell}\right)=\left[\phi_{m 1}\left(z_{\ell}\right) \phi_{m 2}\left(z_{\ell}\right) \mid \theta_{m}\left(z_{\ell}\right)\right]^{T}
$$

With this choice of parameters (modal coefficients) the augmented state equations for the $m$-th mode become

$$
\begin{aligned}
\phi_{m 1}\left(z_{\ell}\right) & =\phi_{m 2}\left(z_{\ell-1}\right)+w_{m 1}\left(z_{\ell-1}\right) \\
\phi_{m 2}\left(z_{\ell}\right) & =-\phi_{m 1}\left(z_{\ell-1}\right)+\left(2-\triangle z_{\ell}^{2} \kappa_{z}^{2}(m)\right) \phi_{m 2}\left(z_{\ell-1}\right) \\
& +w_{m 2}\left(z_{\ell-1}\right) \\
\theta_{m}\left(z_{\ell}\right) & =\theta_{m}\left(z_{\ell-1}\right)+w_{\theta_{m}}\left(z_{\ell-1}\right)
\end{aligned}
$$

where we have selected a random walk model $\left(\dot{\theta}_{m}(z)=\right.$ $\left.w_{\theta_{m}}(z)\right)$ to capture the variations of the modal coefficients with additive, zero-mean, Gaussian noise of covariance $R_{w_{\theta_{m}} w_{\theta_{m}}}$.
Note that when we augment the unknown parameters into the state vector to construct our parametrically adaptive processor, then we assume that they are random (walks) with our pre-computed initial values specified (initial conditions or means) and their corresponding covariances used to bound their uncertainty ( $2 \sigma$ confidence bounds). In fact, if we know more about how they evolve dynamically or we would like to constrain their values, we can place "hard" limits directly into the processor. For instance, suppose we know that the modes are not evanescent; therefore, we can limit the corresponding wave number coefficient excursions practically to $\left(\theta_{\min }, \theta_{\max }\right)$ to avoid these erroneous values by using an augmented parameter model such as:

$$
\theta\left(z_{\ell+1}\right)=\theta\left(z_{\ell}\right), \quad \theta_{\min }<\theta\left(z_{\ell}\right)<\theta_{\max }
$$

thereby constraining any excursions to remain within this interval.

Of course, the random walk model certainly can provide "soft" constraints in the simulation, since the parameter is modeled as Gauss-Markov implying that $95 \%$ of the samples must lie within confidence limits controlled by $\left( \pm 1.96 \sigma_{m, m}\right)$. This constitutes a soft statistical constraint of the parameter variations. However, this approach does not guarantee that the parametrically adaptive processor will remain within this bound; therefore, hard constraints may offer a better alternative. For our runs, we use the implied soft statistical constraint and choose to start the processor with initial parameter estimates close to those values other researchers have meticulously estimated from this data set [28,30,31].

More succinctly, we can write

$$
\Phi_{m}\left(z_{\ell}\right)=\mathbf{A}_{m}\left(z_{\ell-1}\right) \Phi_{m}\left(z_{\ell-1}\right)+\mathbf{w}_{m}\left(z_{\ell-1}\right)
$$

for

$$
\mathbf{A}_{m}\left(z_{\ell-1}\right)=\left[\begin{array}{ccc|c}
0 & 1 & \mid & 0 \\
-1 & 2-\triangle z_{\ell-1}^{2} \kappa_{z}^{2}(m) & \mid & 0 \\
- & - & - & \\
0 & 0 & \mid & 1
\end{array}\right]
$$

The corresponding measurement model is given by

$$
p\left(r_{s}, z_{\ell}\right)=\sum_{m=1}^{M} \theta_{m}\left(z_{\ell}\right) \phi_{m}\left(z_{\ell}\right)+v\left(z_{\ell}\right) ; \quad \ell=1, \cdots, L
$$

with dispersion (sound-speed)

$$
c\left(z_{\ell}\right)=\frac{\omega}{\sqrt{\kappa_{z}^{2}(m)+\kappa_{r}^{2}(m)}}, m=1, \cdots, M ; \ell=1, \cdots, L
$$

This completes the section on the discrete state-space representation of the shallow ocean acoustic (normal-mode) propagation model that is embedded as a "forward propagator" into the subsequent processors for signal enhancement. Note that the initial model parameters are obtained from the prior solution of the boundary value problem as shown in Fig. 1 . 


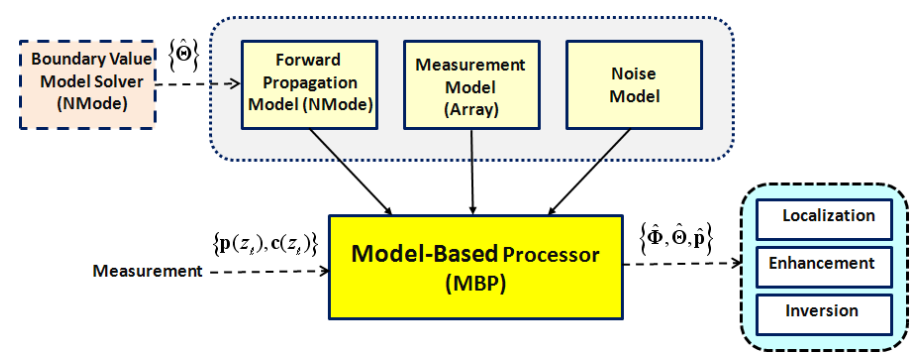

Fig. 1. Model-based processor design: (a) Boundary Solver for initial parameters. (b) Propagator, measurement and noise models. (c) BP. (d) Applications: localization, enhancement (tracking) and inversion.

\section{PROCESSORS}

In this section we briefly develop the processors for our problem with details available in [23]. The basic adaptive problem we pursue in this paper can now be defined in terms of our mathematical models as:

GIVEN a set of noisy pressure-field and sound speed measurements varying in depth, $\left[\left\{p\left(r_{s}, z_{\ell}\right)\right\},\left\{c\left(z_{\ell}\right)\right\}\right]$ along with the underlying state-space model of Eqs. 18, 19 and 20 with unknown modal coefficients, FIND the "best" (minimum error variance) estimate of the modal functions, that is, $\left\{\hat{\phi}_{m}\left(z_{\ell} \mid z_{\ell}\right)\right\},\left\{\hat{\theta}_{m}\left(z_{\ell} \mid z_{\ell}\right)\right\} ; m=1, \cdots, M$ and measurements (enhanced) $\left\{\hat{p}\left(r_{s}, z_{\ell}\right)\right\}$.

We will primarily focus on the particle filter processor, since the unscented Kalman filter has been discussed elsewhere [17],[23] in detail. Note that the UKF is an alternative to the nonlinear or extended Kalman filter processor applied successfully in many of the model-based ocean acoustic applications [1]-[15]. Like the EKF it is still restricted to a unimodal distribution (single peak), but that distribution need not be Gaussian. It also performs a linearization (statistical), but not of the system dynamical model, but of an inherent nonlinear vector transformation requiring "sigma points" which deterministically characterize the underlying unimodal distribution. These points have been pre-calculated for the Gaussian case [17]. It has been shown that the UKF clearly outperforms the $E K F$ and its variants (iterated $E K F$, higher order EKFs, etc.) and is more accurate and precise besides being much easier to implement, since Jacobian matrices are no longer required.

Note also that for the EKF/UKF the underlying posterior distribution has already been decided to be unimodal (multivariate Gaussian), that is,

$$
\begin{aligned}
& \hat{p}\left[\Phi\left(z_{\ell}\right) \mid P_{z}\right]=(2 \pi)^{-N_{\Phi} / 2}\left|R_{\Phi \Phi}\left(z_{\ell} \mid z_{\ell}\right)\right|^{-1 / 2} \times \\
& \exp \left\{-\frac{1}{2}\left(\mathbf{\Phi}\left(z_{\ell}\right)-\hat{\mathbf{\Phi}}\left(z_{\ell} \mid z_{\ell}\right)\right)^{T} R_{\Phi \Phi}^{-1}\left(z_{\ell} \mid z_{\ell}\right) \times\right. \\
& \left.\left(\boldsymbol{\Phi}\left(z_{\ell}\right)-\hat{\boldsymbol{\Phi}}\left(z_{\ell} \mid z_{\ell}\right)\right)\right\}
\end{aligned}
$$

where $\hat{\mathbf{\Phi}}\left(z_{\ell} \mid z_{\ell}\right)$ is the augmented conditional state vector (above) at depth $z_{\ell}$ and $R_{\Phi \Phi}\left(z_{\ell} \mid z_{\ell}\right)$ is the conditional state covariance based on pressure-field measurements up to depth $z_{\ell}$.

A particle filter is a completely different approach to nonlinear filtering in that it removes the restriction of additive Gaussian noise sources and is clearly capable of characterizing multimodal distributions. In fact, it might be easier to think of the $P F$ as a histogram or kernel density like estimator in the sense that it is an empirical probability mass function (PMF) that approximates the desired posterior distribution such that statistical inferences can easily be performed and statistics extracted directly. Here the idea is a radical change in thinking where we attempt to develop an empirical estimation of the posterior distribution following a purely Bayesian approach using Monte Carlo (MC) sampling theory as its enabling foundation. As one might expect the computational burden of the $P F$ is much higher that of $\mathrm{KF}$, since it must provide an estimate of the underlying state posterior distribution component-bycomponent at each $z_{\ell}$-step along with the fact that the number of samples to characterize the distribution is equal to the number of particles.

$$
\hat{\operatorname{Pr}}\left[\phi\left(z_{\ell}\right) \mid P_{z}\right]=\sum_{i=1}^{N_{p}} \mathcal{W}_{i}\left(z_{\ell}\right) \delta\left(\phi\left(z_{\ell}\right)-\phi_{i}\left(z_{\ell}\right)\right) \quad \forall z_{\ell}
$$

$$
\begin{array}{cl}
W_{i}\left(z_{\ell}\right) & \propto \hat{\operatorname{Pr}}\left[\phi_{i}\left(z_{\ell}\right) \mid P_{z}\right] \text { is the estimated weights at depth } z_{\ell} \\
\phi_{i}\left(z_{\ell}\right) & \text { is the } i \text {-th particle at depth } z_{\ell} ; \\
\hat{\operatorname{Pr}}[\cdot] & \text { is the estimated empirical distribution; } \\
P_{z} & \text { is the set of batch pressure-field measurements, } \\
& P_{z}=\left\{p\left(r_{s}, z_{1}\right) \cdots p\left(r_{s}, z_{L}\right)\right\} .
\end{array}
$$

Thus, we see that once the underlying posterior is available, the estimates of important statistics can be extracted directly. For instance, the maximum a posteriori (MAP) estimate is simply found by locating a particular particle $\hat{\phi}_{i}\left(z_{\ell}\right)$ corresponding to the maximum of the PMF, that is,

$$
\hat{\Phi}_{i}^{M A P}(z)=\max _{i} \hat{\operatorname{Pr}}\left[\phi_{i}\left(z_{\ell}\right) \mid P_{z}\right]
$$

while the conditional mean or equivalently the minimum mean-squared error (MMSE) estimate is calculated by integrating the posterior as:

$\hat{\Phi}_{i}^{M M S E}(z)=\int \phi_{i}(z) \hat{\operatorname{Pr}}\left[\phi_{i}\left(z_{\ell}\right) \mid P_{z}\right] d z \approx \frac{1}{N_{p}} \sum_{i=1}^{N_{p}} \mathcal{W}_{i}\left(z_{\ell}\right) \phi_{i}\left(z_{\ell}\right)$

There are a variety of $P F$ algorithms available, but perhaps the simplest is the bootstrap technique [23] which we apply to our problem. The $P F$ design for our problem using the bootstrap approach requires the conditional state transition probability, $\operatorname{Pr}\left[\Phi\left(z_{\ell}\right) \mid \Phi\left(z_{\ell-1}\right)\right]$, and the likelihood (probability) $\operatorname{Pr}\left[p\left(r_{s}, z_{\ell}\right) \mid \Phi\left(z_{\ell}\right)\right]$. Here the state transition is characterized by the underlying augmented state-space model for each mode of Eq. 18, that is, 


$$
\Phi_{m}\left(z_{\ell}\right)=\mathbf{A}_{m}\left(z_{\ell-1}\right) \Phi_{m}\left(z_{\ell-1}\right)+\mathbf{w}_{m}\left(z_{\ell-1}\right)
$$

or

$$
\begin{aligned}
{\left[\begin{array}{c}
\Phi_{m}\left(z_{\ell}\right) \\
--- \\
\theta_{m}\left(z_{\ell}\right)
\end{array}\right]=} & {\left[\begin{array}{ccc}
A_{m}\left(z_{\ell}\right) & \mid & 0 \\
- & - & - \\
0 & \mid & 1
\end{array}\right]\left[\begin{array}{c}
\Phi_{m}\left(z_{\ell-1}\right) \\
--- \\
\theta_{m}\left(z_{\ell-1}\right)
\end{array}\right] } \\
+ & {\left[\begin{array}{c}
W_{m}\left(z_{\ell-1}\right) \\
--- \\
W_{\theta_{m}}\left(z_{\ell-1}\right)
\end{array}\right] }
\end{aligned}
$$

where $W_{m} \sim \mathcal{N}\left(0, R_{W_{m} W_{m}}\right), W_{\theta_{m}} \sim \mathcal{N}\left(0, R_{W_{\theta_{m}} W_{\theta_{m}}}\right)$, $\phi_{m}(0) \sim \mathcal{N}\left(\bar{\phi}_{m}(0), R_{\phi_{m} \phi_{m}}\right), \theta_{m}(0) \sim \mathcal{N}\left(\bar{\theta}_{m}(0), R_{\theta_{m} \theta_{m}}\right)$.

and therefore (under the Gaussian assumption), we have that

$$
\begin{aligned}
& \operatorname{Pr}\left[\Phi\left(z_{\ell}\right) \mid \Phi\left(z_{\ell-1}\right)\right]=(2 \pi)^{-N_{\Phi} / 2}\left|P_{m}\left(z_{\ell}\right)\right|^{-1 / 2} \times \\
& \exp \left\{-\frac{1}{2}\left(\Phi\left(z_{\ell}\right)-\mathbf{A}_{m}\left(z_{\ell-1}\right) \Phi_{m}\left(z_{\ell-1}\right)\right)^{T} P_{m}\left(z_{\ell}\right)^{-1} \times\right. \\
& \left.\left(\Phi\left(z_{\ell}\right)-\mathbf{A}_{m}\left(z_{\ell-1}\right) \Phi_{m}\left(z_{\ell-1}\right)\right)\right\}
\end{aligned}
$$

with the state covariance given by $P_{m}\left(z_{\ell}\right)=$ $\mathbf{A}_{m}\left(z_{\ell-1}\right)^{T} P_{m}\left(z_{\ell-1}\right) \mathbf{A}_{m}\left(z_{\ell-1}\right)+R_{W_{m} W_{m}}$.

For the bootstrap implementation, we need only draw noise samples from the state and parameters distributions and use the dynamic models above (normal-mode/random walk) in Eq. 18 to generate the set of particles, $\left\{\Phi_{m i}\left(z_{\ell}\right)\right\}$ for each $i=$ $1, \cdots, N_{p}$.

The likelihood, on the other hand, is determined from the nonlinear pressure-field measurement model of Eq. 19, that is, for each mode we have

$$
p_{m i}\left(r_{s}, z_{\ell}\right):=\theta_{m i}\left(z_{\ell}\right) \phi_{m_{i}}\left(z_{\ell}\right)+v\left(z_{\ell}\right), \text { for } \ell=1, \cdots, L
$$

and therefore the scalar likelihood (assuming Gaussian noise) is

$$
\begin{aligned}
& \operatorname{Pr}\left[p\left(r_{s}, z_{\ell}\right) \mid \Phi\left(z_{\ell}\right)\right]=\frac{1}{\sqrt{2 \pi R_{v v}}} \times \\
& \exp \left\{-\frac{1}{2 R_{v v}}\left(p\left(r_{s}, z_{\ell}\right)-\sum_{m=1}^{M} \theta_{m i}\left(z_{\ell}\right) \phi_{m 1}\left(z_{\ell} ; i\right)\right)^{2}\right\}
\end{aligned}
$$

Thus, we estimate the posterior distribution using a sequential Monte Carlo approach and construct a bootstrap particle filter [10] using the following steps:

- Initialize: $\Phi_{m}(0), w_{z_{\ell}} \sim \mathcal{N}\left(0, R_{w w}\right), W_{i}(0)=1 / N_{p} ; i=$ $1, \cdots, N_{p}$

- $\underline{\text { State Transition: }} \Phi_{m}\left(z_{\ell}\right)=\mathbf{A}_{m}\left(z_{\ell-1}\right) \Phi_{m}\left(z_{\ell-1}\right)+$ $\mathbf{w}_{m}\left(z_{\ell-1}\right)$;

- Likelihood Probability: $\operatorname{Pr}\left[p\left(r_{s}, z_{\ell}\right) \mid \Phi\left(z_{\ell}\right)\right]$ of Eq. 28;

- Weights: $W_{i}\left(z_{\ell}\right)=W_{i}\left(z_{\ell-1}\right) \times \operatorname{Pr}\left[\Phi_{m}\left(z_{\ell}\right) \mid \Phi_{m}\left(z_{\ell-1}\right)\right]$; 


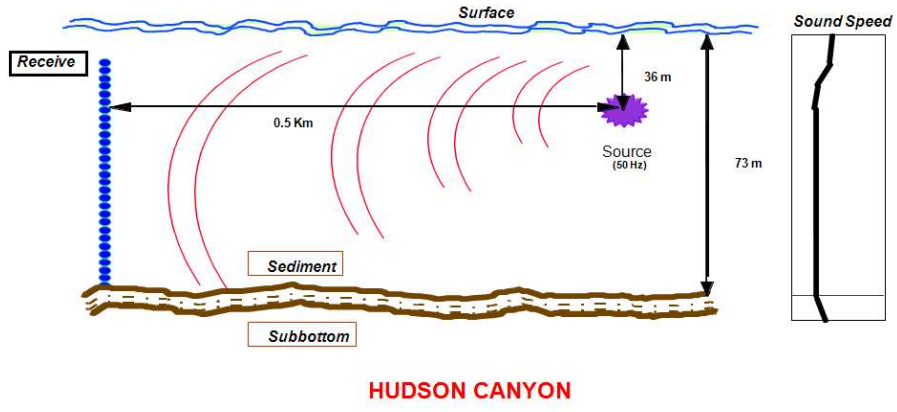

Fig. 3. Hudson Canyon experiment geometry and structure: (a) Source at $36 \mathrm{~m}$ depth and $0.5 \mathrm{Km}$ range, $50 \mathrm{~Hz}$. (b) 23 -element vertical hydrophone array. (c) 5 modes support the water column.

Coring project borehole 6010 . The seismic and coring data are combined with sediment properties measured at that site. Excellent agreement was determined between the model and data indicating a well-known, well-documented shallow water experiment with bottom interaction and yielding ideal data sets for investigating the applicability of a $B P$ to measured ocean acoustic data [11], [12]. The experiment was performed at low frequencies $(50-600 \mathrm{~Hz})$ in shallow water of $73 \mathrm{~m}$ depth during a period of calm sea state as shown in Fig. 3. A calibrated acoustic source was towed at roughly $36 \mathrm{~m}$ depth along the $73 \mathrm{~m}$ isobath radially to distances of 4 to $26 \mathrm{Km}$. The ship speed was between 2 and $4 \mathrm{Kts}$. The fixed vertical hydrophone array consisted of 24 phones spaced $2.5 \mathrm{~m}$ apart extending from the seafloor up to a depth of about $14 \mathrm{~m}$ below the surface. CTD and SSP measurements were made at regular intervals and the data were collected under carefully controlled conditions in the ocean environment. The normalized horizontal wave number spectrum for a $50 \mathrm{~Hz}$ temporal frequency is dominated by 5 modes occurring at wave numbers between 0.14 to 0.21 $m^{-1}$ with relative amplitudes increasing with increased wave number. A SNAP [13] simulation was performed and the results agree quite closely, indicating a well-understood ocean environment.

In order to construct the state-space propagator, we require the set of parameters which were obtained from the experimental measurements and processing (wave number spectra). The horizontal wave number spectra were estimated using synthetic aperture processing [11]. Eight temporal frequencies were employed: four on the inbounds $(75 \mathrm{~Hz}, 275 \mathrm{~Hz}, 575 \mathrm{~Hz}, 600 \mathrm{~Hz})$ and four on the outbound $(50 \mathrm{~Hz}, 175 \mathrm{~Hz}, 375 \mathrm{~Hz}, 425 \mathrm{~Hz})$. In this application we will confine our investigation to the $50 \mathrm{~Hz}$ case, which is well-documented, and to horizontal ranges from $0.5-4 \mathrm{Km}$. The raw measured data was processed (sampled, corrected, filtered, etc.) and supplied for this investigation.

\section{A. Adaptive PF Design}

The design and development of the environmentally adaptive $P F$ proceeds through the following steps: (1) preprocessing the raw experimental data; (2) solving the boundary value problem $(B V P)$ [9] to obtain initial parameter sets for each temporal frequency (e.g. wavenumbers, modal coeffi-

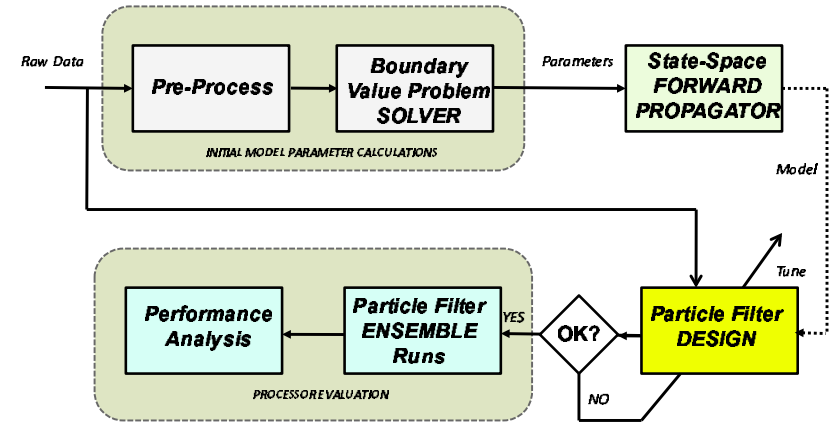

Fig. 4. $\quad P F$ design/development procedure: (a) Initial parameters/conditions. (b) Design runs. (c) Ensemble runs.

cients, initial conditions, etc.); (3) state-space forward propagator simulation of synthetic data for $P F$ analysis/design; (4) application to measured data; and (5)PF performance analysis as shown in Fig. 4.

Pre-processing of the measured pressure-field data follows the usual pattern of filtering, outlier removal and Fourier transforming to obtain the complex pressure-field as a function of depth along the array. This data along with experimental conditions (frequencies, sound-speed profiles (CTD measurements), boundary conditions, horizontal wavenumber estimators (see [12] for details) provide the input to the normal mode $B V P$ solutions (SNAP [6], KRACKEN [7], etc.) yielding the output parameters. These parameters are then used as input to the state-space forward propagator (see Fig. 4) developed in Sec. II.

The state-space propagator is then used to develop a set of synthetic pressure-field data with higher resolution than the original raw data (e.g. 46-element array rather than 23-element at half-wave inter-element spacing). This set represents the "truth" data that can be investigated when "tuning" the $P F$ (e.g. number of particles, covariances, etc.). Once tuned, the processors are applied directly to the measured pressure-field data (23-elements) after re-adjusting some of the processor parameters (covariances). Here the performance metrics are estimated and processor performance analyzed. Since each run of the $P F$ is a random realization, that is, the process noise inputs are random, an ensemble of results are estimated with ensemble statistics presented. In this way, we can achieve a detailed analysis of the processor performance prior to fielding and operational version. In this paper we constrain our discussion results to processing the noisy experimental pressure-field measurements [12] as compared to a previous paper [24] that concentrated on the synthetic data set generated from the forward propagator. 


\section{B. Results}

First we investigate the enhancement capabilities of the $P F$ in estimating the pressure-field over a 100-member ensemble shown in Fig. 5. Here we see the raw data (dotted blue line) as well as both maximum a-posteriori (MAP) estimates (solid red line) and conditional mean $(C M)$ estimates (dotted magenta line with circles). Both estimators appear to track the field quite well until some erratic measurements near the bottom of the channel. The predictions appear more reasonable than the noisy data. The corresponding innovations (residual) sequence is also shown. Classically, both estimators produced satisfactory zero-mean/statistical whiteness tests $(Z-M / W-T$ : $6.2 \times 10^{-4}<4.9 \times 10^{-1} / 6.3 \%$ as well as the WSSR tests indicating a "tuned" processor [17]. The UKF processor also produced reasonable results for the enhanced pressure-field.

Ensemble mode tracking results are shown in Figs. 6 and 7 for each of the modal function estimators, the $P F(M A P / C M)$ and the UKF. In Fig. 6 we observe that the performance of the $P F(M A P / C M)$ appears to track the modes quite well especially compared to the $U K F$. The $P F$ estimators perform equivalently. Two of the modal function estimates (first two) exhibit the largest errors as shown in Fig. 7 while the final three functional estimates are much better. It is interesting to note that the modal coefficient estimates are constantly being adapted (adjusted) by the processor throughout the runs attesting to the nonstationary nature of the ocean statistics as illustrated in Fig. 8.

We also illustrate the multimodal aspect of the oceanic data by observing the modal function posterior PDF estimates for modes 1 and 5 as illustrated in Fig. 9. It is clear from the plots that for each depth multiple peaks appear in the posterior estimates. The pressure-field posterior is better behaved almost producing a near unimodal posterior for the predicted field. Visualizing a peak at each depth produces a "smooth" estimate (MAP) as shown in Fig. 10. This completes the analysis of the Hudson Canyon experimental data and the $P F$ processing performance.

\section{SUMMARY}

In this paper we have developed on-line model-based solutions to the ocean acoustic signal processing problem based on the normal-mode propagation model and a vertical sensor array measurement system. We have demonstrated that a parametrically adaptive particle filter is able to track the modal functions while jointly adjusting environmental parameters (modal coefficients) in a variable shallow ocean. The algorithms employed were the unscented Kalman filter and the particle filter both modern approaches applied to this problem. We compared their performances and found better performance of the $P F$ over a 100-member ensemble. Next we plan to investigate the adaptivity to the wave number parameters - a more sensitive set to environmental changes. Much more effort must be applied to gain a full understanding of applying these approaches to usual ocean acoustic problems (localization, tracking, inversion, etc.). Our future efforts will be focused on extending the processors to those problems.

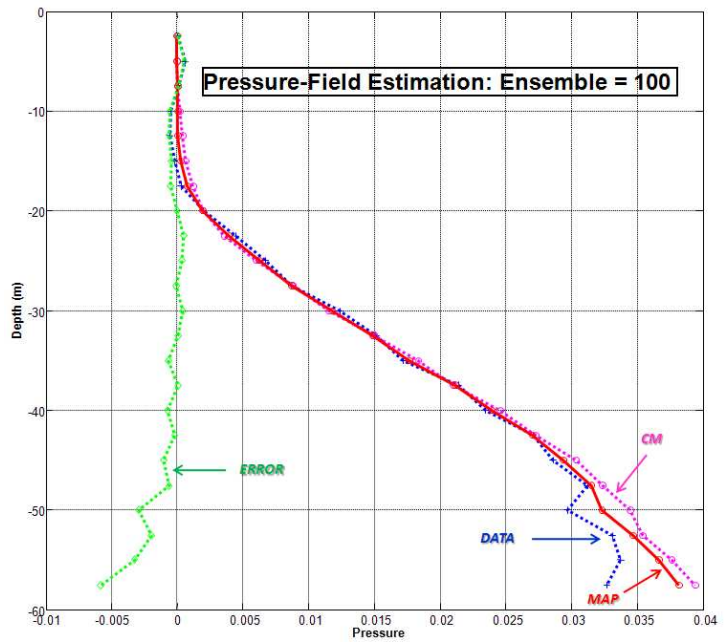

Fig. 5. Raw/enhanced pressure-field (blue dots) data from the Hudson Canyon experiment using particle filter estimators: MAP (red), conditional mean $(C M)$ in magenta and the $U K F$ (turquoise) with corresponding innovations (residuals) sequence (green).

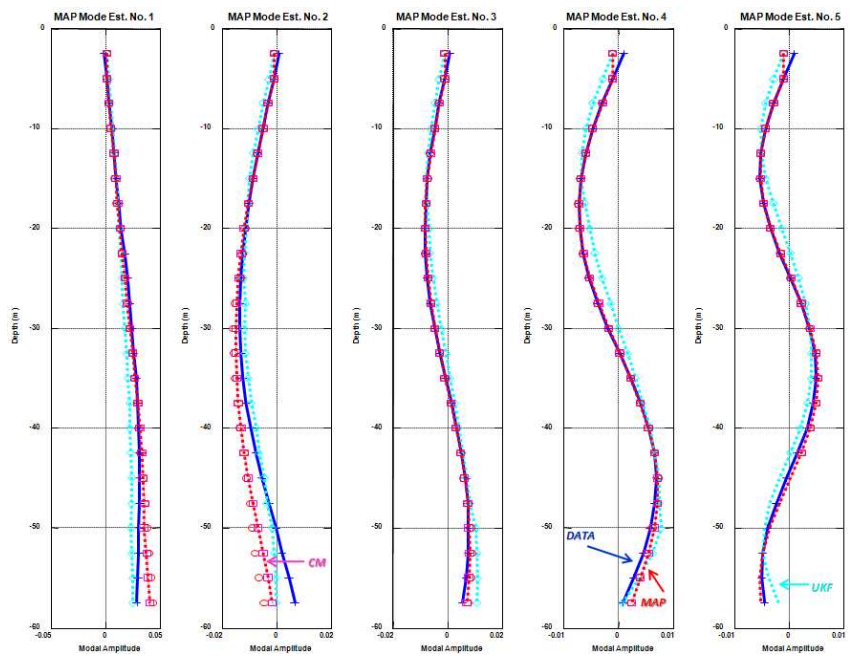

Fig. 6. Modal function tracking (estimation): Raw experimental data (blue plus), $U K F$ (turquoise dots), MAP (red circles) and $C M$ (magenta squares) particle filters.
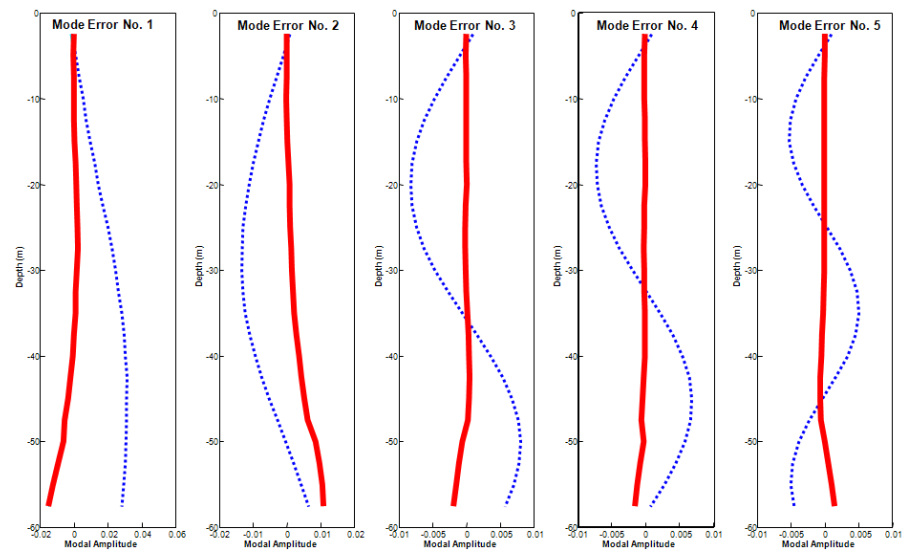

Fig. 7. Modal function tracking errors: Raw experimental data (blue plus) and MAP (red circles) particle filters errors. 

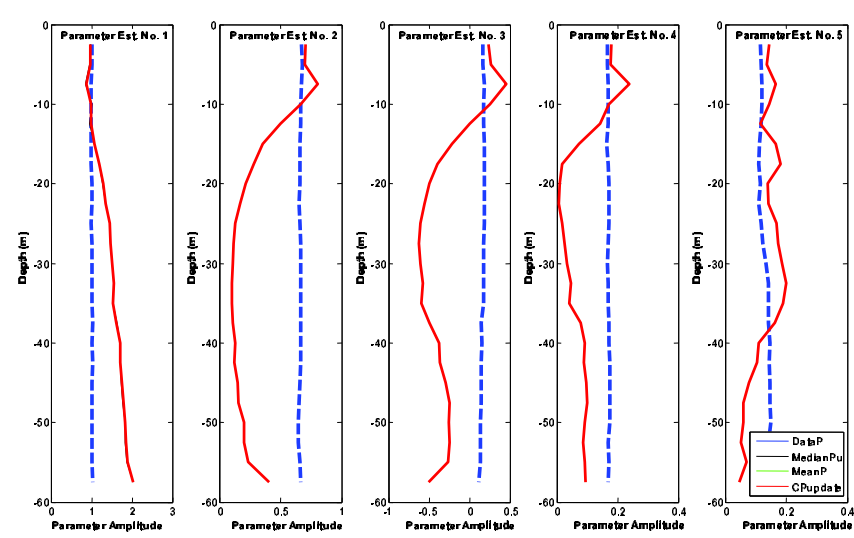

Fig. 8. Adaptive modal coefficient parameter estimation data from the Hudson Canyon experiment using the MAP (red) particle filter.
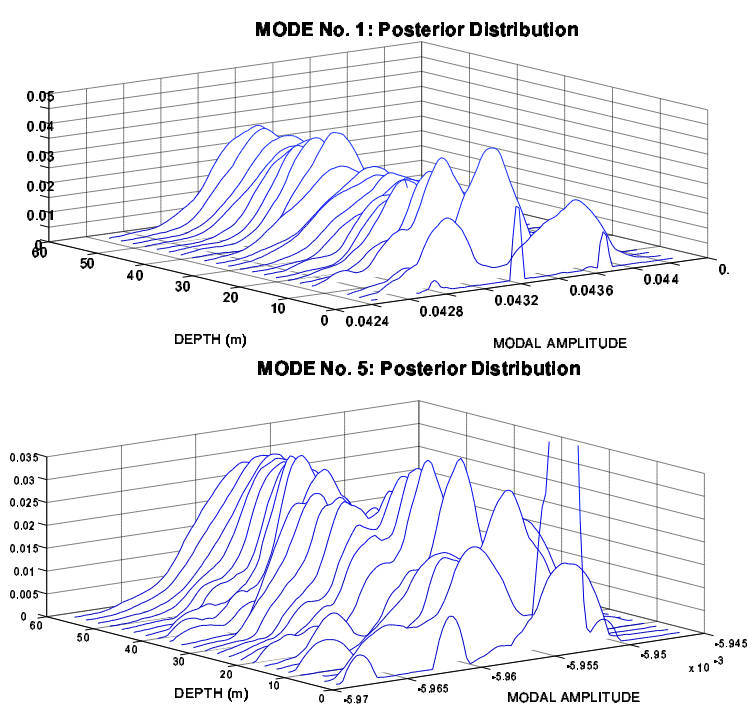

Fig. 9. PMF posterior estimation (modes 1 and 5) surfaces for experimental Hudson Canyon data (particle vs. time vs. probability).

\section{ACKNOWLEDGEMENTS}

This article has been authored by Lawrence Livermore National Security, LLC under Contract No. DE-AC5207NA27344 with the U. S. Department of Energy. Accordingly, the United States Government retains and the publisher, by accepting the article for publication, acknowledges that the United States government retains a non-exclusive, paidup, irrevocable, world-wide license to publish or reproduce the published form of this article or allow others to do so, for United States Government purposes.

\section{REFERENCES}

[1] M. J. Hinich, "Maximum likelihood signal processing for a vertical array," J. Acoust. Soc. Am., 54, 499-503, 1973.

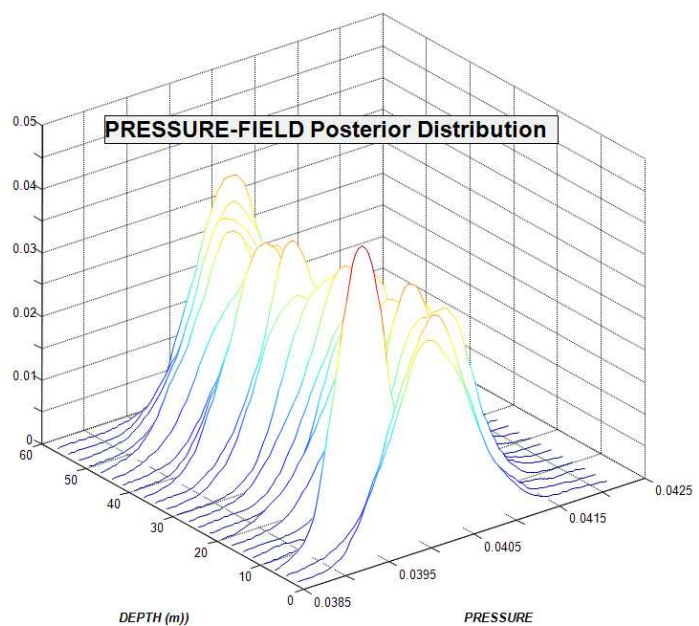

Fig. 10. Pressure-field posterior PMF estimation surface for experimental Hudson Canyon data (particle vs. time vs. probability.

[2] C. S. Clay, "Use of arrays for acoustic transmission in a noisy ocean," Res. Geophys., 4, (4), 475-507, 1966.

[3] H. P. Bucker, "Use of calculated sound fields and matched-field detection to locate sound in shallow water," J. Acoust. Soc. Am., 59, 329-337, 1976.

[4] A. M. Richardson, and L. W. Note, "A posteriori probability source localization in an uncertain sound speed, deep ocean environment," $J$. Acoust. Soc. Am., 89, (6), 2280-2284, 1991.

[5] E. J. Sullivan and D. Middleton, "Estimation and detection issues in matched-field processing", IEEE J. Oceanic Eng., 18, (3), 156-167, 1993.

[6] F. B. Jensen and M. C. Ferla, "SNAP: the SACLANTCEN normal-model propagation model," Report SM-121, Italy: SACLANTCEN, 1979.

[7] M. B. Porter, "The KRACKEN normal mode program," Report SM-245, Italy: SACLANTCEN, 1991.

[8] H. Schmidt, "SAFARI: Seismo-acoustic fast field algorithm for range independent environments," Report SM-245, Italy: SACLANTCEN, 1987.

[9] F. B. Jensen, Kuperman, W. A., Porter M. B.,and H. Schmidt, Computational Ocean Acoustics New York: Amer. Instit. Physics Press, 1994.

[10] J. V. Candy and E.J. Sullivan. "Model-based processor design for a shallow water ocean acoustic experiment", J. Acoust. Soc. Am., 95, (4) 2038-2051, 1994.

[11] W. M. Carey, J. Doutt, R. Evans and L. Dillman, "Shallow water transmission measurements taken on the New Jersey continental shelf," IEEE J. Oceanic Eng., 20, (4), 321-336, 1995.

[12] A. R. Rogers, Y. Yamamoto and W. Carey, "Experimental investigation of sediment effect on acoustic wave propagation in shallow water," $J$. Acoust. Soc. Am., 93,1747-1761, 1993.

[13] F. B. Jensen, and M.C. Ferla, "SNAP: the SACLANTCEN normal-mode acoustic propagation model," SACLANTCEN Report,

[14] C. S. Clay, and H. Medwin, Acoustical Oceanography. New York:Wiley, 1977.

[15] J. V. Candy and E. J. Sullivan. "Ocean acoustic signal processing: a model-based approach.” J. Acoust. Soc. Am., 92, (12), 3185-3201, 1992.

[16] A. Jazwinski, Stochastic Processes and Filtering Theory. New York:Academic Press, 1970.

[17] J. V. Candy, Model-Based Signal Processing. Hoboken, N.J.:Wiley/IEEE Press, 2006.

[18] B. Ristic, S. Arulampalam and N. Gordon, Beyond the Kalman Filter: Particle Filters for Tracking Applications, Boston: Artech House, 2004.

[19] O. Cappe, E. Moulines and T. Ryden, Inference in Hidden Markov Models, New York: Springer-Verlag, 2005.

[20] S. Godsill and P. Djuric, "Special Issue: Monte Carlo methods for statistical signal processing." IEEE Trans. Signal Proc., vol. 50, 2002.

[21] P. Djuric, J. Kotecha, J. Zhang, Y. Huang, T. Ghirmai, M. Bugallo and J. Miguez, "Particle Filtering," IEEE Signal Proc. Mag. vol. 20, No. 5, 19-38, 2003.

[22] S. Haykin and N. de Freitas, "Special Issue: Sequential state estimation: 
from Kalman filters to particle filters." Proc. IEEE, vol. 92, No. 3, 399574, 2004.

[23] J. V. Candy, Bayesian Signal Processing: Classical, Modern and Particle Filtering Methods. Hoboken, N.J.:Wiley/IEEE Press, 2009.

[24] J. V. Candy, "An adaptive particle filtering approach to tracking modes in a varying shallow ocean environment" Proc. OCEANS'11, Santander, Spain, 2011. 